\title{
La difficile émergence d'une cohérence en didactique des langues par l'éclectisme théorique et la multiplicité de conflits d'interprétation
}

Yvon Rolland

\section{(2) OpenEdition}

\section{Journals}

Édition électronique

URL : http://journals.openedition.org/asp/912

DOI : 10.4000/asp.912

ISBN : 978-2-8218-0398-5

ISSN : 2108-6354

\section{Éditeur}

Groupe d'étude et de recherche en anglais de spécialité

\section{Édition imprimée}

Date de publication : 1 décembre 2004

Pagination : 127-144

ISSN : 1246-8185

Référence électronique

Yvon Rolland, "La difficile émergence d'une cohérence en didactique des langues par l'éclectisme théorique et la multiplicité de conflits d'interprétation », ASp [En ligne], 45-46 | 2004, mis en ligne le 02 mars 2010, consulté le 06 mai 2019. URL : http://journals.openedition.org/asp/912 ; DOI : 10.4000/ asp. 912

Ce document a été généré automatiquement le 6 mai 2019.

Tous droits réservés 


\title{
La difficile émergence d'une cohérence en didactique des langues par l'éclectisme théorique et la multiplicité de conflits d'interprétation
}

\author{
Yvon Rolland
}

1 Cet article fait suite à des travaux de recherche qualitative, descriptive et expérimentale en didactique de l'anglais relevant de l'apprentissage phonologique, l'apport des livrets d'histoires et de la poésie, l'intérêt des nouvelles technologies, l'impact de l'inconscient cognitif, comme l'influence de l'affectif. La difficulté d'un chercheur en didactique des langues est de réussir à faire émerger une problématique de recherche cohérente face à ce qui relève souvent soit de prises de position dogmatiques fondées sur telle école de pensée, soit d'une absence gênante de certitude généralisée, soit d'émergences de pratiques se souciant peu de théorie. Une fois le sujet de recherche délimité, le premier obstacle provient d'un éventail de domaines de références multiples souvent très éloignés les uns des autres. Le risque est alors soit d'atomiser l'analyse, soit d'arriver à des synthèses négligeant l'imbrication, la complémentarité, voire l'antinomie de tels ou tels paramètres. La didactique des langues relève d'un champ scientifique non unifié qui se traduit par un éclectisme théorique aussi déroutant qu'incontournable : c'est ce que nous nous efforcerons de démontrer dans un premier temps. Les théories psychologiques ont enrichi, mais aussi directement influencé ce vaste champ scientifique, les théories d'apprentissage sont en conséquence variées et apportent toutes des principes intéressants. Les théories linguistiques ont toutes marqué la didactique des langues. Mais ces fondements appartiennent à des écoles de pensées divergentes engendrant inévitablement des conflits d'interprétation multiples et inhibiteurs. La notion d'âge pour l'apprentissage, l'apport de la psychanalyse, le recours à l'imaginaire, l'apprentissage et ses modalités diverses, le concept même de phonèmes, les conflits linguistiques sont autant de domaines aux facettes contradictoires problématiques : c'est ce que nous nous 
attacherons à analyser dans un deuxième temps. Enfin, l'atteinte d'une plus grande cohérence en didactique ne passerait-elle pas par la nécessaire transcendance de champs scientifiques encore peu exploités? Et les pratiques sur le terrain ne peuvent-elles pas englober nécessairement une juxtaposition quelque peu équilibriste d'approches théoriques divergentes, mais aussi infiniment complémentaires? Nous terminerons cette réflexion par une tentative de réponses à ces deux questions.

\section{L'éclectisme théorique d'un champ scientifique non unifié}

2 La multiplicité des apports théoriques est certes déroutante à première vue, mais elle est aussi le gage d'une richesse de fondements qui s'avèrent souvent incontournables, car remettant en cause des concepts pouvant poser problème.

\subsection{Le volet psychologique}

3 Le volet psychologique a directement façonné les orientations théoriques de l'apprentissage. La psychologie béhavioriste a tout d'abord incarné la psychologie du comportement et du conditionnement opérant. Le sujet apprend grâce à une modification du comportement provoqué par des stimuli venant de l'environnement. La linguistique y ajoutera le distributionnalisme structurel pour aboutir au structuralisme linguistique.

4 La «Gestalttheorie » et les psychologues de la forme insistent ensuite sur les limites de simples conditionnements et précisent que la compréhension de schèmes d'action complexes est primordiale. On avance alors la notion de compréhension.

La psychologie cognitiviste va détrôner ces théories psychologiques en mettant en avant la perception, la mémoire, les représentations mentales et la résolution de problèmes. La théorie cognitive vise à élaborer une théorie de la connaissance ; l'organisme possèderait des structures adaptatives, constituées de systèmes actifs de réponses et de réorganisation (Gaonac'h 1987 : 118). Deux notions importantes seraient à intégrer dans cette perspective, «l'interactionnisme » ou l'interaction permanente entre le sujet et le milieu, et « le constructivisme » ou l'action de l'organisme sur le milieu, les résistances de celui-ci, avec une succession d'équilibres et de perturbations représentant des paliers et des stades. Le développement cognitif et le développement linguistique seraient liés selon Piaget (Gaonac'h 1987: 119), de même que la composante sociale qui ne doit pas être oubliée selon les psychologues cognitivistes Bandura, Vygotsky ou Bruner. Les constructivistes ne reconnaissent pas le bien-fondé des travaux expérimentaux de Chomsky qui ont porté sur la réalité psychologique des transformations et sur le rôle de la structure syntaxique profonde dans le traitement du langage. La psychologie cognitive demeure incontournable dans la compréhension de la didactique des langues, mais si le concept est pertinent, il serait perfectible. Le développement cognitif des individus ne serait pas le même selon le domaine de connaissances considéré, les capacités de mémorisation ne seraient pas les mêmes selon le niveau de connaissances auxquelles elles s'appliquent, l'évolution de la personnalité ne serait pas déterminée par les aspects cognitifs, l'évolution cognitive dépendrait beaucoup plus de la personnalité qu'on ne le croyait au début, précisent Hureau et Loarer (1995: 150). Pour l'anglais de spécialité, le domaine de connaissances spécifique abordé prend appui sur une maturité cognitive 
certainement plus grande. Mais en est-il de même pour la dimension linguistique ? Sans doute que non. Ajoutons que les formes d'apprentissage sont intentionnelles (de type constructiviste) ou non (relevant alors du conditionnement - Berbaum 1992). Nous mesurons déjà les difficultés engendrées par la psychologie cognitiviste pure et dure. Ces fondements psychologiques sont liés à l'apprentissage.

\subsection{Les théories d'apprentissage}

6 Les théories d'apprentissage sont le reflet de tel ou tel courant psychologique et, selon les époques, s'avèrent plus ou moins privilégiées, puis par la suite décalées. Il ressort que tous les fondements liés à une théorie précise ne sauraient forcément être écartés pour autant, engendrant ainsi des conflits problématiques.

7 Si le behaviorisme fut la panacée pendant plusieurs décennies, c'était parce qu'il « faisait passer l'acquisition des langues étrangères par le montage systématique d'habitudes verbales acquises par réitération» (Galisson $1980: 7-8$ ). Selon Skinner, deux principes fondamentaux étaient à considérer : le sujet devait produire une réponse à un moment donné, et celle-ci devait être renforcée immédiatement. Deux "contingences de renforcement " sont à la base de tout comportement : elles correspondent à l'interaction entre trois classes d'événements successifs, une stimulation (production d'une réponse), un comportement de l'organisme (réponse), et un événement extérieur à l'organisme, qui suit le comportement. Si les aspects formels du langage sont privilégiés, le comportement dans l'environnement physique et social est également inséré (Gaonac'h 1987 : 25).

La théorie médiationnelle ajouta d'autres aspects que ceux directement observables : les phénomènes implicites, internes ou mentaux. Réactions affectives et manifestations physiologiques sont provoquées par les stimuli verbaux. Cette approche néo-béhavioriste a apporté des éléments intéressants pour le contexte d'acquisition et a contribué à remettre en question des situations d'apprentissage trop structurées.

Les théories nativistes prennent appui sur les propriétés biologiques innées. Chomsky défend l'innéisme et un ensemble fini de règles formelles constituant une grammaire générative permettant d'engendrer toutes les phrases correctes de cette langue. Apprendre une langue « est lié à un ensemble de principes linguistiques inné, un système inné d'acquisition («Language Acquisition Device») permettant d'intégrer les données linguistiques primaires " (Narcy 1990: 56-57). Chomsky a eu une grande influence dans l'acquisition linguistique et le rôle de la structure syntaxique profonde dans le traitement $\mathrm{du}$ langage. Ces fondements théoriques ont contribué à favoriser une réaction antibehavioriste irréversible.

Les théories environnementalistes réfutent l'innéisme et partent du principe que l'expérience et les forces environnementales sont capitales dans l'acquisition d'une langue. L'hypothèse de pidginisation et le processus de fossilisation relèvent de créations de systèmes linguistiques indépendants de ceux de la langue cible; ce phénomène dit de nativisation s'oppose à celui de dénativisation pour le sujet qui doit reconstruire son système linguistique pour arriver à être en conformité avec la langue cible (Andersen in Ellis 1994 : 379). Le modèle d'acculturation de Schumann (Larsen-Freeman \& Long 1991 : 253) évoque certaines variables externes à l'apprenant; Brown parle de processus d'acculturation et a identifié quatre stades que sont « l'euphorie, le choc culturel, le stress culturel et l'adaptation à la nouvelle culture » (Brown in Ellis 1985 : 251). L'intérêt de 
telles approches est d'évoquer des paramètres sociaux et psychologiques, certes intéressants mais difficiles aussi à mesurer exactement.

11 D'autres théories prennent en compte innéisme et environnementalisme. Il s'agit d'approches dites «interactionnistes». Prenant appui sur la psycholinguistique expérimentale et la psychologie cognitive, elles expliquent que la syntaxe émane des propriétés du discours humain et que les changements syntaxiques sont opérés par des principes psycholinguistiques et pragmatiques liés à la perception de la parole et à la production en interaction réciproque entre deux sujets. L'acquisition supposerait un passage d'un mode pragmatique de communication à un mode syntaxique. L'atout de ces approches est de mettre en avant la communication et de considérer la langue comme un outil au service de la communication, ce qui représente indubitablement une avancée non négligeable pour l'apprentissage d'une langue vivante. Mais nous mesurons déjà la difficile adéquation entre environnementalisme et innéisme pourtant à la base de l'interactionnisme.

12 L'approche heuristique "procède par la découverte des faits par l'apprenant lui-même » (Narcy 1990 : 268). Ces travaux s'intègrent à la psychologie cognitive. La perception et la mémoire sont des éléments importants. On ne veut plus ignorer les déterminants contextuels et situationnels de la signification; les données linguistiques, sémantiques, contextuelles sont liées au système cognitif et au processus de mémorisation.

13 Nous mesurons la richesse de ces théories qui ont permis de faire avancer la didactique institutionnelle qui recommande la reproduction, la contextualisation, la communication et la réflexion sur la langue, qui sont des émanations des théories pré-citées.

\subsection{Les théories linguistiques}

Les théories linguistiques ont enrichi la didactique des langues, mais elles sont aussi source de conflits d'interprétation. L'influence de la linguistique structurale a été manifeste pendant des années et, contre toute attente, n'a pas entièrement disparu, même si l'âge d'or des approches audio-orales ou audio-visuelles est révolu. Les théories behavioristes de Skinner trouvent écho chez Bloomfield, linguiste du distributionnalisme structural s'intéressant à la distribution des éléments constituants de la phrase selon deux axes; l'axe syntagmatique, déroulement linéaire de l'énoncé, permettra des exercices de transformation syntaxique, et l'axe paradigmatique favorisera des exercices de manipulations mécaniques de substitution sur un axe vertical applicable à chacun des éléments constituants de la phrase. L'approche structurale naît de ces exercices structuraux aux régularités combinatoires des structures de base. Les modèles proposés sont simples à imiter et à répéter. La répétition intensive doit favoriser des réflexes et des automatismes, car elle est fondée sur un travail mécanique contrôlé, répété aussi souvent que ce sera nécessaire pour atteindre une fixation en mémoire profonde équivalente à une véritable imprégnation. On propose à l'apprenant une phrase stimulus et un modèle de réponse, puis d'autres. Ainsi naît l'approche audio-orale insistant tout particulièrement sur les distinctions phonologiques propres au code oral. Si les exercices structuraux sont écartés des recommandations didactiques actuelles, cette approche est encore déterminante dans l'élaboration des progressions, l'accent est mis sur l'acquisition de structures, c'est-à-dire de schémas morpho-syntaxiques. L'approche audio-visuelle arrive dans la droite ligne de l'audio-oral : automatismes par un recours au renforcement immédiat plutôt qu'explications grammaticales et utilisation massive de sketches 
dialogués, de supports sonores et visuels avec un écrit introduit après une phase solide d'imprégnation orale. On retrouve de nombreux points communs avec ce qui se passe toujours dans les formations actuelles. Le but des exercices structuraux ou de fixation était d'induire une généralisation portant sur l'enchaînement de mots selon une structure déterminée. On apprend l'analogie de structure, induite par la transposition d'éléments dont la similitude des uns par rapport aux autres tient à leur disposition dans la structure (Gaonac'h 1987 : 27). On y voit le moyen d'enseigner de manière inductive la grammaire sans avoir besoin de l'expliciter; la grammaire va donc pouvoir être analysée et s'apprendre de manière implicite. Cette approche a favorisé les possibilités de construction d'énoncés grammaticaux complexes, de même que la compétence linguistique pure fondée sur des énoncés grammaticaux corrects. L'erreur est enfin reconnue comme passage obligé de l'apprentissage. L'approche visuelle renforcée des documents est aussi reconnue comme facilitatrice pour la compréhension et la mémorisation des outils linguistiques. Ce sont autant de facteurs que l'on retrouve toujours dans les cursus.

15 Enfin, l'approche structure-globale audio-visuelle se réfère à la "Gestalt Psychologie » et entend par «situation" les attitudes, les besoins et les capacités de l'apprenant. Le problème de l'apprentissage devient celui de la perception. Apprendre une langue "nécessite de sélectionner les éléments pertinents sur le plan phonologique, morphémique et syntaxique » (Gaonac'h $1987:$ 78). Des termes comme "présentation, explication, répétition, exploitation" sont utilisés et restent d'actualité dans de nombreux ouvrages encore en vigueur de nos jours. On intègre aussi la dimension paralinguistique comme la situation d'interlocution ou la situation spatio-temporelle. On prend en compte la globalité des activités, non seulement au plan linguistique, mais aussi au plan des acquisitions liées aux conduites reliées au langage. On parle d'activité structurante pour l'apprenant avec en phonologie, par exemple, des tâches prenant en compte simultanément prosodie, intonation et rythme. Les structures sont amenées par une situation simulée par des moyens audio-visuels. Le sens global de l'énoncé est relié à la structure employée pour rendre l'activité mentale structurante. Cette globalité se retrouve en phonologie avec la notion d'opposition ou de traits distinctifs de Jakobson et Halle cités par Gaonac'h (1987:63). On ne définit plus les phonèmes selon des propriétés communes, mais selon des différences permettant de les opposer aux autres : l'analyse en traits distinctifs fut déterminante en didactique des langues avec l'émergence d'exercices de phonétique axés sur la discrimination de phonèmes. Les activités phonologiques actuelles ne sont pas très éloignées des exercices structuraux d'antan.

Plus récemment, la méthode verbo-tonale va encore plus loin en tentant de rééduquer l'apprenant sourd aux sons étrangers en prenant en compte l'être humain dans sa totalité. La théorie du crible phonologique de Troubetzkoy (1986 [1949] : 54) repose sur le constat que l'apprenant s'approprie le système de sa langue et, en entendant la langue étrangère, il emploiera involontairement le crible phonologique correspondant à sa langue maternelle, ce qui l'induira à l'erreur. Cette notion de rééducation est nouvelle pour ce qui est de l'apprentissage d'une langue étrangère et ceci met l'accent sur ce qui pose souvent problème, la nativisation.

17 La linguistique structurale a fortement influencé et influence toujours plus ou moins la didactique des langues. Mais les limites de ces méthodes se sont vite avérées gênantes : la pertinence des énoncés en contexte n'était pas toujours assurée et l'objectif communicatif rarement atteint; la communication, bien qu'en ligne de mire, était empêchée par des 
activités contraintes non communicatives menant difficilement à un début d'autonomie (Coste in O'Neil 1993 : 101) ; si l'accumulation de structures grammaticales était l'objectif majeur, les dimensions lexicale, phonologique et sémantique étaient oubliées.

Avec les fonctions de communication et l'approche communicative, les linguistes sont passés de la linguistique de la langue à la linguistique de la parole (Heddesheimer \& Lagarde 1978 in O'Neil 1993 : 103). Les mots-clés ne sont plus « code, système, structure linguistique, compétence linguistique ", mais "énonciation, discours, acte de parole, compétence de communication ». Moirand précise que la communication est un :

échange interactionnel entre au moins deux individus situés socialement, échange qui se réalise au travers de l'utilisation de signes verbaux et non verbaux, chaque individu pouvant être tour à tour soit producteur soit consommateur de messages. (Moirand 1982 in O'Neil 1993 : 104)

20 C'est aussi « la transmission d'une information avec trois paramètres que sont le message, l'émetteur, le récepteur" (Julié 1994: 176). Les quatre composantes de la capacité de communication que sont compréhension et expression orales et écrites servent une pédagogie de l'authenticité. Widdowson insiste sur l'utilisation d'énoncés dans des « actes de communication » (1979: 53). Sont inventoriés les éléments essentiels pour atteindre un niveau seuil de connaissance linguistique permettant de faire face aux besoins fondamentaux communicatifs. Ces besoins langagiers trouvent leur réponse dans des situations de communication effective : celui qui questionne ne sait pas la réponse. Cette approche semble plus logique par rapport à la démarche intellectuelle de l'apprenant, car c'est le besoin langagier qui déclenche le processus d'apprentissage de la structure et non l'inverse (penser à une structure et inventer un besoin de communication pour utiliser celle-ci). La notion d'apprentissage centré sur l'apprenant revient en force au détriment de l'enseignement trop centré sur l'enseignant. L'anglais de spécialité proposé dans le second degré technologique et professionnel, ainsi que dans l'enseignement supérieur pour spécialistes d'autres disciplines a su tirer profit de ces nouvelles données.

L'âge d'or des pratiques notionnelles-fonctionnelles semble pourtant un peu révolu au vu des dernières orientations institutionnelles. Plusieurs raisons semblent émerger aujourd'hui. La progression des difficultés grammaticales n'était-elle pas négligée ? La dimension phonologique n'était-elle pas trop écartée, posant évidemment problème pour la compréhension de la communication dite authentique? Les besoins langagiers d'adolescents sont-ils aisément identifiables, notamment par rapport à ceux des adultes, pour lesquels cette approche a été initialement conçue? Ces besoins langagiers ne se présentaient-ils pas sous la forme d'inventaires approximatifs? L'appel au cognitif ne faisait-il pas cruellement défaut? N'en résulte-t-il pas un concept très proche des guides de conversation qui proposent des formules toutes faites au touriste novice? De nombreux manuels des années 1980 annonçaient un habillage notionnel-fonctionnel pour des contenus souvent structuraux. Bailly $(1998: 40)$ n'hésite pas à parler du " communicatif de première génération », qui, malgré ses apparences de rupture avec le structuralisme, restait de conception behavioriste : les problèmes d'apprentissage étaient de toutes façons résolus par le simple contact avec la langue suivi d'un ajout " plaqué » de quelques exercices structuraux mécaniques et décontextualisés.

D'où l'apparition dans les années 1990 d'un communicatif de deuxième génération. Une dimension méthodologique d'obédience cognitiviste dans la gestion mentale des activités de l'apprenant, incluant gradualité, accompagnement et aides multiples, est introduite ; parallèlement, la dimension linguistique revient en force avec la grammaire et la 
phonologie ; la communication, certes difficile en cours de langue, ne peut exister sans apport linguistique; inversement, un apport linguistique ne peut se faire sans tenir compte de la dimension de communication. L'apport linguistique prend appui sur l'approche énonciative qui vient à la rescousse d'une approche communicative dépourvue de métalinguistique. Il s'agit là d'une activité consciente contrairement à l'épilinguistique qui relève de l'inconscient. L'apprenant est ainsi amené à suivre un processus déductif et / ou inductif de découverte progressive et parfois intuitive du fonctionnement de la langue dans ce l'on appelle "pratique raisonnée de la langue ». Car il est admis qu'un enseignement axé sur la communication ne saurait se dispenser de la compréhension du fonctionnement de la langue cible.

La notion d'éclectisme théorique trouve ainsi son sens: les théories psychologiques ont engendré des théories d'apprentissage, elles-mêmes inspirées de l'apport de théories linguistiques multiples et très antinomiques. La didactique des langues, dans ses fondements théoriques relevant d'un champ scientifique vaste et non unifié, peine à trouver ses marques : les conséquences sur la recherche sont inévitables, créant autant de conflits d'interprétation qu'il y a d'approches théoriques et mettant le chercheur en difficulté dans ses travaux pour cerner sa problématique avec cohérence.

Tous ces apports théoriques divergents apportent des fondements antinomiques que sont conditionnement et innéisme d'une part, expérience et cognitivisme d'autre part. Aspects linguistiques formels peu pertinents, habillage fonctionnel dépourvu de cognitif, méthodologie cognitiviste et métalangue énonciative inhibant la communication, la cohérence didactique devient une gageure. La recherche dévoile aussi de nouveaux conflits d'interprétation au niveau de concepts, incontournables pourtant, comme l'âge, la psychanalyse, l'imaginaire ou le phonème. La notion d'apprentissage s'en trouve complexifiée.

\section{La multiplicité de conflits d'interprétation au niveau d'apports théoriques divergents}

L'apprentissage prend appui sur des concepts posant déjà problème. Un facteur qui semble avoir toujours alimenté les débats des chercheurs en apprentissage des langues est celui de l'âge.

\subsection{L'âge critique de réceptivité}

Des positions divergentes (Singleton 1989: 101 - 117) sont formulées par les chercheurs. La première stipule que l'acquisition serait favorisée pour un apprenant jeune, qui, au delà de douze ou quinze ans, perdrait plus difficilement son accent maternel. La seconde approche met en avant l'âge plus avancé comme un atout. Des adolescents apprendraient autant en cinq ans que d'autres, plus jeunes, en onze. La troisième position insiste sur les avantages d'un démarrage très précoce. Enfin, une dernière recherche précise tout l'intérêt d'un apprentissage précoce à très long terme. L'intensité et le temps d'exposition, l'immersion ou l'instruction sont par exemple des données faussant toute tentative de synthèse, dans la mesure où l'on peut être amené à comparer des expériences reposant sur des critères finalement différents. En fait, une analyse très fine des paramètres retenus pour chaque expérience s'impose pour tenter de résoudre une 
partie des conflits d'interprétation inhérents à cette notion d'âge : il est vrai que les aptitudes à la communication et à la dimension phonologique semblent meilleures lorsqu'on est jeune; par opposition, les aptitudes cognitives en rapport avec la conceptualisation syntaxique seraient mieux exploitées par des sujets plus âgés (Snow, Hoefnagel-Höhle \& Cummins 1979 in Singleton 1989: 113). Il ressort que les apports théoriques retenus orienteront les résultats : la linguistique axée sur la phonologie pour les plus jeunes, d'une part, et celle fondée sur la morpho-syntaxe, le cognitivisme pour les plus âgés d'autre part, aboutiront à des conflits d'interprétation gênants. La notion d'âge pose donc problème et les fondements retenus conditionnent déjà les réponses.

D'autres fondements semblent source de conflits pour la recherche. C'est le cas de la psychanalyse.

\subsection{La psychanalyse}

28 La psychanalyse est pourtant utile pour tenter de mieux comprendre certains mécanismes. L'identification de certains apprenants à leur professeur en est un exemple. Stengel (1939), Kris (1952), Guiora (1972) et Taylor (1974), cités par Rolland (2003: 61 - 64), se sont penchés sur ce problème; mais si leur obédience freudienne les unit, leurs interprétations divergent: l'infantilisation liée à l'apprentissage, la flexibilité d'un ego qui se développe, la naissance d'une nouvelle identité, le narcissisme provoquant des blocages se télescopent et ne rendent pas la tâche du chercheur aisée. Mais le rôle de l'enseignant n'est-il pas systématiquement omis ? La relation cognitiviste élève/étudiantsavoir est actuellement valorisée sur le plan théorique. Mais la relation entre l'enseignant et le savoir conditionne la première sur un plan pratique, et enfin la relation enseignantélève/étudiant de type psychanalytique influe inévitablement sur les résultats. L'approche psychanalytique ressurgirait lorsque la vague cognitiviste sera épuisée, déclare Houssaye (1992 : 117). Cette dernière est largement négligée dans la recherche en didactique et pourtant la dimension parfois fusionnelle ou au contraire rédhibitoire entre l'enseignant et son élève/étudiant ne peut pas être niée et influence le reste. Mais cette dimension est bien sûr très difficile à cerner, voire impossible. Elle est sans doute occultée pour cette raison.

29 Un autre conflit survient lorsque l'on se penche sur le problème de la contextualisation. Si les didacticiens s'accordent à prendre en compte celle-ci pour de meilleures conditions d'apprentissage, le rôle de l'imaginaire est sujet à controverse.

\subsection{Le rôle de l'imaginaire}

30 Le choix des matériaux est souvent celui de thèmes et de contextes proches du vécu des apprenants, ce qui semble indispensable si l'on ne veut pas décourager complètement ces derniers d'apprendre. Les écoliers (à cause de leur âge), les collégiens et les lycéens (en période d'adolescence) auraient besoin de prendre appui sur un contexte qui leur est familier pour rendre la tâche plus facile. Mais le danger ne serait-il pas de proposer des situations d'apprentissage trop réalistes peu attractives ? C'est pourtant ce que font tous les manuels scolaires de l'école au lycée. On écarte tout ce qui pourrait «ennuyer » le public scolaire, parce que trop éloigné de ses préoccupations premières. La nourriture à l'école, le sport au collège, l'environnement au lycée sont des thèmes très répandus. «Dans la plupart des ensembles pédagogiques, on reste souvent en présence d'un 
quotidien terre à terre agrémenté d'un habillage un peu fantaisiste » (O'Neil 1993 : 157). Or la notion d'imaginaire est néanmoins capitale pour des scientifiques comme Piaget (1987 [1964] : 104-197). Le manque d'abstraction des jeunes apprenants s'explique par une conscience de soi qui ne se construit que progressivement, l'animisme, la notion de vie révélant autant de confusion liée à l'immaturité. Freud (1982 [1938] : 194-195) parle de l'importance du rêve pour un apprenant qui se construit à partir de son enfance :

le rêve favorise une régression vers l'état infantile et l'inconscient de la vie

psychique n'est autre que la phase infantile de cette vie.

31 Jung (1964: 135) insiste aussi sur la nécessaire intégration de matériaux initiatiques comme les « contes, par exemple, exprimant directement les processus psychiques de l'inconscient collectif ». Nous le voyons, la notion de contextualisation révèle un conflit d'interprétation. Un difficile équilibre ne doit-il pas être trouvé ? Trop de réalisme semble dommageable. O'Neil (1993: 157) dit à juste titre qu'à côté

d'éléments réalistes répondant à la curiosité des élèves pour les pays dont on étudie la langue, il faut faire une place réelle au rêve et à la fantaisie.

Les directives institutionnelles successives et nombreuses se révèlent aussi déroutantes parfois. Elles réorientent les circulaires pour compenser des dérives observées chemin faisant. Le meilleur exemple reste l'apprentissage phonologique.

\subsection{L'apprentissage phonologique}

L'apprentissage phonologique, inclus dans les années 1960-70 lors de l'âge d'or des méthodes structurales, fut écarté à l'arrivée de l'approche communicative, et réintroduit depuis, de sorte que toutes les dernières instructions de l'école, du collège et du lycée concernant l'anglais, font mention d'un impressionnant volet phonologique que d'autres langues ignorent (l'allemand, l'espagnol). C'est ainsi que l'on mentionne à nouveau les paires minimales, pourtant oubliées pendant près de vingt ans. Ces contradictions sont préjudiciables. Mais le plus délicat est que le concept même de phonème pose problème et reste source de multiples conflits d'interprétation.

Hyman (1975: 60-97) analyse ces interprétations diverses. La première (années 1930, École de Prague) considère le phonème comme une unité phonologique distinctive : «le phonème est la plus petite unité distinctive de la langue » (Bloomfield in Troubetzkoy 1986 [1949] : 3 - 11). C'est le concept de « traits distinctifs » ou marques de différenciation phoniques particulières; par exemple les consonnes sont perçues selon des traits distincts comme le voisement, la nasalité, la stridence, la durée et le lieu d'articulation. Les travaux de Troubetzkoy visaient à saisir les contrastes au niveau du voisement des consonnes et de l'acuité des voyelles, ceux de Jakobson introduisaient une présentation binaire des traits phonologiques ; les propriétés acoustiques sont autant utilisées que les propriétés articulatoires. Cette approche intéressante du phonème comme unité distinctive reste complexe pour une transposition didactique.

La deuxième interprétation (années 1940-50) considère le phonème comme une réalité phonétique physique sur la base de propriétés distributionnelles. Jones (Hyman 1975 : 60) définit le phonème comme « un son important de la langue relié à d'autres sons prenant place dans des séquences sonores particulières ». Gleason (Hyman $1975: 60)$ ajoute qu'il s'agit «d'une classe de sons phonétiquement identiques faisant preuve de certains schémas caractéristiques de 'distribution' dans la langue ». Les paires minimales présentent ainsi deux phonèmes différents : une unité minimale de son sera susceptible 
de faire une différence de sens, telle serait la première définition du phonème. La substitution permet le contraste minimal qui n'est pas fiable à cent pour cent, car dans le cas de distribution défective (cas des phonèmes /h/ /zj/), les phonèmes ne peuvent pas être mis en contraste. "Un phonème serait une classe de phonèmes similaires en distribution complémentaire" (Lass 1984: 20). Les notions de "graphème» et de " morphème » ne rendent pas la tâche aisée pour l'apprenant et cette approche physique, si elle clarifie chaque représentation, ne résout pas les risques de confusion entre phonie et graphie. Cette approche est encore largement plébiscitée dans les recommandations institutionnelles.

La troisième interprétation part du phonème comme réalité psychologique. C'est la position des mentalistes que les linguistes de l'École de Prague réfutent. Pour de Courtenay, cité par Twadell (Hyman 1975: 72), «le phonème est une réalité mentale évoquant l'intention du locuteur ou l'impression du locuteur ou les deux ». Tous les théoriciens s'accordent à penser que le système phonémique d'une langue a un effet comportemental sur son utilisateur et joue un rôle dans la perception des sons d'une langue étrangère. La théorie du filtre phonologique de Troubetzkoy avance la difficulté d'analyser de manière pertinente les sons étrangers dans la mesure où, inconsciemment, nous les percevons au travers des sons maternels. Tout est une question de conscience linguistique fortement imprégnée par la langue 1. La phonologie générative part de l'innéisme et d'une entité psychologique supposant une pré-programmation basée sur une richesse d'universaux innés. Ceci prend en compte la dimension inconsciente difficile à cerner, nous l'avons déjà vu, mais qu'il faut peut-être ne pas négliger pour autant. Ce débat fut déjà le nôtre pour la psycholinguistique et la psychanalyse. Il pose le problème de la forme linguistique et de son utilisation par un individu donné.

Ce conflit d'interprétation phonétique rejoint celui des théories linguistiques, dont nous avons parlé. Les différentes théories linguistiques, comme les théories d'apprentissage s'avèrent contradictoires, nous l'avons souligné. La dimension linguistique même pose problème.

\subsection{La dimension linguistique}

Les approches linguistiques pré-citées ont toutes laissé des marques dans les orientations institutionnelles: l'apprentissage phonologique se fait encore selon les principes structuralistes, puisque les activités de discrimination phonétique portant sur les paires minimales par exemple sont reconnues et recommandées. L'apprentissage morphosyntaxique se fait autant par le biais de situations de communication et des notionsfonctions qui y sont liées, et donc de l'approche communicative, que de la pratique raisonnée de la langue issue de l'approche linguistique énonciative. Or les deux approches semblent antinomiques: communiquer n'est pas conceptualiser. Les limites de ces approches respectives doivent être aussi compensées par l'approche cognitive: l'apprenant doit à tout moment comprendre ce qu'il fait et pourquoi. Comment le chercheur et l'enseignant peuvent-ils raisonnablement et logiquement intégrer tout ceci dans leur démarche? Ne ressemblent-ils pas à des jongleurs équilibristes? Un minimum de linguistique structurale est requis pour les savoirs formels, une approche communicative de deuxième génération d'obédience cognitiviste est préconisée pour les savoir-faire linguistiques et méthodologiques, tandis qu'une approche énonciative est nécessaire pour la conceptualisation. La dimension linguistique est très dépendante 
d'écoles de pensée très opposées les unes aux autres: ceci ne facilite pas l'émergence d'une logique didactique cohérente.

\subsection{Le concept d'apprentissage}

Le concept d'apprentissage est lui aussi, rappelons-le, difficile à cerner, compte tenu des théories dont nous nous sommes fait écho. Si les théories interactionnistes sont considérées comme importantes pour l'institution, les théories constructivistes le sont aussi ; toutes deux relèvent pourtant de la psychologie cognitive. La communication est un maître-mot, mais la méthodologie est importante et la dimension linguistique ne peut être occultée. Le behaviorisme semble sérieusement remis en question, de même que les théories dites nativistes, car trop orientées sur l'étude formelle de la langue; il reste néanmoins des vestiges de ces approches. La langue, bien qu'abordée en situation de communication est aussi répertoriée de manière formelle; il suffit de compulser les référentiels (Instructions officielles de collège, de lycée) pour s'en convaincre: structures, lexique et blocs lexicalisés, phonologie et grammaire sont classés dans des tableaux structurés. Apprendre une langue ne signifie-t-il pas tendre à en maîtriser sa forme? Certains manuels trahissent d'ailleurs ce problème en habillant la démarche de façon fonctionnelle et communicative en apparence, mais avec un fond qui rappelle à bien des égards la dimension structurale d'antan. Il semble difficile de faire cohabiter la communication qui suppose une progression non programmable, construite à partir des besoins langagiers du public, une méthodologie qui implique une progression fonctionnelle et linguistique en spirales (mêmes fonctions de communication pour chaque classe, mais structures de plus en plus élaborées d'année en année) et des référentiels précis, structurés et cadrés pour chaque niveau d'enseignement par exemple. L'approche communicative suppose d'autre part un minimum de spontanéité, ce que l'approche heuristique ne peut proposer. Comment résoudre ces conflits? À savoir la communication, les savoirs linguistiques et culturels, et la méthodologie peu compatibles. Comment communiquer, maitriser les formes, dominer des concepts linguistiques et culturels, développer des stratégies méthodologiques en un ensemble cohérent? Le problème est que la psychologie cognitive est elle-même problématique, qu'elle bénéficie d'un effet de mode inéluctable, et qu'elle implique deux notions, interactionnisme et constructivisme ; elle n'a détrôné qu'en partie seulement les théories nativistes (innéisme et acquisition formelle de la langue), car les théories interactionnistes intègrent innéisme, mais aussi environnementalisme et cognitivisme. Et les théories constructivistes n'intègrent pas toujours la dimension sociale qui faisait défaut aux études piagétiennes, notamment. La psychologie cognitive n'apporte pas toutes les réponses attendues dans la mesure où elle néglige l'apprenant et le contexte d'apprentissage.

Nous le voyons, les théories d'apprentissage pré-citées sont en partie retenues et en partie tronquées. Les conflits d'interprétation sont ainsi inévitables. Chaque approche a ses limites qu'il est souhaitable de ne pas dépasser pour éviter toute dérive. Arriver à un subtil dosage, pratiquer telle orientation en sachant que des limites sont à prévoir semblent indispensable pour ne pas discréditer ces fondements théoriques sur lesquels l'institution prend appui.

41 La cohérence didactique ne peut être obtenue à partir d'une multiplicité de conflits d'interprétation déstabilisateurs. Ne serait-il pas indispensable de transcender ce champ 
scientifique non unifié pour tenter de faire émerger une plus grande cohérence didactique?

\section{La nécessité de transcender pour une plus grande cohérence didactique}

L'élargissement à de nouveaux champs théoriques ne permettrait-il pas d'arriver à un meilleur équilibre? Narcy $(1997: 55)$ propose une transcendance de diverses théories qui, au-delà d'un éclectisme souvent contradictoire, permettrait de mieux intégrer la composante sociale, comportementale et neuroscientifique dans un champ scientifique non encore unifié. Si la psychanalyse et la psycholinguistique apportent des données incontournables, mais souvent ignorées, voire écartées, de nouveaux fondements scientifiques comme l'éthologie et la neurobiologie peuvent ouvrir de nouvelles perspectives.

\subsection{Les éthologues}

Les éthologues se penchent sur l'étude des comportements et leur analyse de l'expérience antérieure est intéressante. Leur analyse est plus convaincante que celle des béhavioristes trop marqués par le conditionnement. Cyrulnik (1983: 65) parle des représentations de notre perception du monde conditionnées par notre personnalité, notre culture et notre langage. Toute perception est conditionnée par qui nous sommes et la façon dont nous ressentons le contexte dans lequel elle se situe. Il y aurait dans certains cas des décalages entre l'appris et l'acquis; Berbaum (1991: 40) parle d'images mentales d'une réalité. Laborit (1994 : 114 - 121) avance l'idée que la personnalité d'un apprenant est forgée par la pérennité de schémas corporels dans le temps, et de l'utilisation du langage comportant des processus associatifs véhiculant des images mentales ancrées en chacun d'entre nous.

\subsection{Les neurobiologistes}

Les neurobiologistes insistent par ailleurs sur le handicap d'une certaine immaturité cognitive des apprenants dans le système scolaire et supérieur qui est due au développement progressif du néo-cortex lié au raisonnement, et qui doit être compensé par la prise en compte de la puissance du cerveau limbique émotionnel omniprésent chez les jeunes. Une dimension affective bienfaitrice permettrait de mieux structurer le développement cognitif, on parle maintenant d'intelligence émotionnelle, voire de «quotient émotionnel» (Goleman 1997 : 14). Ceci va de pair avec une acuité sensorielle reconnue chez des apprenants adolescents. Intégrer une approche multisensorielle semble nécessaire. Le domaine visuel doit être renforcé, car lorsqu'il est bien exploité, il influe sur la cognition et la mémorisation. Le domaine auditif, bien que source de problèmes en compréhension orale, est aussi très prégnant à long terme, lorsqu'il est associé au ludique : c'est le cas de chansons mémorisées au pouvoir cognitif inestimable. Le domaine kinesthésique inclut l'expression corporelle et le mime : l'approche "TPR » (Asher 1969 : 3-17) est fondée sur le principe de la parole-action et prend ses racines dans les théories comportementalistes. La chronologie compréhension-production utilise le geste et la parole et vise une mémoire d'autant plus active qu'elle est accompagnée de la 
motricité, qui est la base des apprentissages premiers. Les apprenants auraient plus de facilité à transférer les acquis vers d'autres capacités. Pour Varela et al. (1993: 63), la dualité physique et mentale relève d'une expérience vécue impliquant la réunion de l'esprit et du corps : une question posée signifie une réflexion intégrant la personne qui la pose et le processus par lequel elle la pose, ce qui débouche sur une signification nouvelle. La kinesthésie implique aussi la dimension émotionnelle (Trocmé-Fabre 1992). L'impact sur une motivation affective de type intrinsèque (Thill 1997 : 377) est capital, de même que sur la mémoire affective épisodique (Delannoy 1994: 61), ce qui renforcera motivation et mémoire cognitive et aura des prolongements à long terme. Laborit (1994 : 19-20) insiste sur l'importance d'une expérience agréable pour l'apprentissage pour répondre au principe de plaisir et de motivation. La synchronicité sensorielle est aussi utile dans la mesure où « une sensorialité en déclenche une autre, grâce au lien établi par l'affectivité » (Trocmé-Fabre 1992: 79). Les phénomènes d'empathie semblent aussi plus présents chez ce public encore jeune que chez des adultes à la personnalité plus affirmée. "Le cerveau a été conçu pour réagir à l'expression des émotions, ce qui revient à dire que l'empathie est une donnée biologique » (Goleman 1997 : 132-137) et que les apprenants imitent plus facilement ce qu'ils observent. Les charges émotionnelles négatives devraient être évitées en minimisant les facteurs anxiogènes liés à tout apprentissage. Il faut en fait comprendre que la communication actuellement très en vogue en didactique institutionnelle doit intégrer tout ce qui fait la communication, qui n'est pas que verbale ou linguistique, mais aussi surtout non verbale et extra-linguistique : comment se fait-on comprendre à l'étranger si l'on ne parle pas la langue du pays, si ce n'est par le non verbal (gestuelle et mimique)?

Il ressort de cet élargissement théorique que l'éthologie et la neurobiologie permettent de mieux intégrer les comportements, le contexte d'apprentissage, l'intelligence émotionnelle, la multisensorialité et l'empathie. Ces fondements viennent renforcer de façon plus concrète le cognitivisme et le constructivisme enrichis d'une dimension sociale de Vygotsky.

\subsection{Une nécessaire juxtaposition d'approches complémentaires}

La psychologie cognitive pure et dure est actuellement valorisée sur le plan didactique théorique avec les dérives que nous venons d'analyser. On en oublie trop trois facteurs importants comme l'apprenant, véritable entité physique et psychologique, l'objet d'apprentissage, ce qu'il représente et qui va déterminer l'individu dans son attitude, et enfin, la situation d'apprentissage avec son environnement social, familial et humain (Berbaum 1992 : 132).

Apprendre serait une démarche cognitive par le traitement de l'information, une démarche affective par les attitudes développées et une démarche volontaire par l'implication personnelle de l'apprenant. Un équilibre doit prévaloir, comme pour l'approche structuraliste après les années 1960-70, ou l'approche notionnellefonctionnelle après les années 1980.

Il reste que la juxtaposition de ces multiples théories contradictoires et les innombrables conflits d'interprétation qui en découlent ne facilitent pas la tâche du chercheur didacticien qui peine à faire émerger une problématique cohérente en didactique des langues. Il doit tout d'abord partir d'une étude historique qui explique la survivance de certaines pratiques en contradiction avec la vague actuelle. Il devra comprendre 
pourquoi celles-ci subsistent contre vents et marées, en essayant d'être le plus objectif possible ; il faudra notamment se détacher de sa propre expérience d'élève ayant connu telle ou telle orientation théorique. Il faudra ne pas se laisser circonvenir par les effets de mode qui mettent en exergue telle ou telle théorie plus que telle autre, avoir de fait, un esprit critique et tenter de gommer certaines dérives peu visibles, effet de mode oblige. Il faudra enfin tenter d'équilibrer l'ensemble par l'apport de nouveaux champs théoriques.

Ceci suppose une juxtaposition très délicate de fondements retenus pour certains aspects positifs, mais débarrassés de ce qui a justement posé problème à l'expérience dans le temps.

C'est le cas des théories structuralistes entachées de conditionnement, de mécanismes automatiques formels dépourvus de contexte. C'est aussi le cas des notions fonctions de communication entachées de superficialité proche de guides pour touristes pressés et négligeant toute réflexion linguistique. C'est aussi le cas de l'approche communicative de première génération faisant l'impasse sur la méthodologie et des objectifs cohérents, quand n'étaient pas plaqués des exercices structuraux dénués de contexte.

Une juxtaposition équilibrée d'approches contradictoires mais nécessairement complémentaires semblerait ainsi incontournable. Les théories structuralistes se justifient toujours pour certaines activités phonologiques axées sur la forme, pour pratiquer phonèmes et schémas intonatifs; encore faut-il veiller à bien contextualiser les énoncés en situation de communication pour plus de pertinence. $Y$ ajouter l'approche multisensorielle n'en sera que plus bénéfique. Mais veiller à ne pas abuser de celle-ci est aussi important pour éviter de retomber dans un conditionnement inhibiteur. Intégrer ces principes dans une démarche cognitive et communicative sera aussi salutaire.

Les notions fonctions, comme les structures grammaticales doivent servir de repères, mais en aucun cas être traitées dans une approche théorique trop exclusive : les utiliser pour des activités de communication contextualisées en s'assurant d'une bonne maitrise de la forme impliquant par la suite une approche énonciative semble cohérent.

53 La psychologie cognitive axée sur la méthodologie et le traitement de l'information, ne doit pas négliger la communication, la dimension linguistique, culturelle et conceptuelle, ni étouffer l'effet enseignant, la contextualisation et l'apprenant. L'approche énonciative enrichira l'approche communicative par la métalinguistique nécessaire à tout apprentissage pertinent. Tenir compte de l'âge du public pour les exigences affichées sera indispensable: dimension phonologique à privilégier chez les jeunes apprenants et morpho-syntaxe et métalinguistique à approfondir chez ceux qui sont plus âgés. Le rôle de l'enseignant et l'impact qu'il a sur son public, s'il est difficile à mesurer, car souvent instinctif, devra tout de même être pris en compte comme paramètre par les didacticiens. Les situations d'apprentissage devraient en outre porter sur des choix de matériaux ludiques sensés plaire au public visé tout en incluant une dimension intellectuelle, initiatique, éducative et formative enrichissante.

Inclure quand cela est possible le multisensoriel pour y intégrer émotions et mémorisation sera gratifiant. Il semble important aussi d'intégrer les comportements d'apprenants qui ne sont pas des réceptacles de savoirs mais des individus qui réagissent, ressentent, pensent. Les activités de pré-écoute ou de pré-lecture en phase d'anticipation sont primordiales pour amener le public à se positionner face à des indices qu'il lui faudra traiter chemin faisant. Une bonne empathie est ici facilitatrice pour aider l'enseignant à mieux percevoir l'expérience et le vécu du public. 
Nous le voyons, il s'agit vraiment d'une juxtaposition délicate à équilibrer ; arriver à faire coexister environnementalisme, interactionnisme et constructivisme n'est pas chose facile. $Y$ adjoindre le comportementalisme de l'éthologie et l'intelligence émotionnelle élucidée par la neurobiologie rééquilibrera l'ensemble. Mais seule une réflexion poussée permettra de s'assurer de la complémentarité des fondements retenus. Une bonne connaissance de tous ces champs théoriques est indispensable au chercheur didacticien pour lui permettre d'avancer dans ses travaux, mais les écueils dûs aux conflits d'interprétation seront difficiles à éviter.

\section{Conclusion}

56 L'éclectisme théorique, dont nous nous sommes fait écho, provient d'une série de théories psychologiques, de théories d'apprentissage et en conséquence d'approches linguistiques qui se sont succédées et qui révèlent des fondements souvent antinomiques. C'est aussi la notion même d'apprentissage qui a évolué fortement au fil des années: apprendre l'anglais dans les années 1950 relevait de la lecture, de la traduction et de la mémorisation de vocabulaire et de règles grammaticales. Dans les années 1960, les théories structuralistes ont apporté une amélioration pour l'époque : la phonétique était étudiée, et l'audio-visuel venait contextualiser la langue. Les années 1980 virent l'émergence d'un plus grand souci de pertinence dans l'approche linguistique avec les notions fonctions et un recentrage sur l'apprenant. Les années 1990 permirent d'affiner l'approche communicative intégrant la psychologie cognitive : l'ensemble équilibré paraissait enfin satisfaisant : l'élève / étudiant est au centre de son apprentissage, une gestion mentale est effectuée par l'apprenant, une dimension linguistique est réaffirmée avec notamment la phonologie et l'énonciation. La psychologie cognitive, si elle a beaucoup apporté au niveau méthodologique, ne doit pour autant privilégier abusivement un côté du triangle didactique (l'apprenant et le savoir), mais doit aussi intégrer les deux autres côtés que sont l'enseignant et l'apprenant, l'enseignant et le savoir. Tenter d'augmenter le plaisir d'apprendre en injectant des paramètres relatifs aux comportements, à l'expérience, à l'acuité sensorielle et à l'empathie sera précieux. Certes, ces champs théoriques que sont le comportementalisme, les neurosciences en général ne sont pas faciles à analyser, mais ne doivent pas être occultés pour autant.

Carr (1993 : 162) prend justement position par rapport aux réticences de ses pairs, devant le concept de phonème vu comme une réalité psychologique. Sa remarque vaut aussi pour la didactique en général et sa délicate dimension humaine. Il compare ce conflit à «la terre tournant autour du soleil, nous ne pouvons la voir tourner, mais nous pouvons voir les conséquences de ce mouvement, ce qui revient à dire que de nombreuses théories ne sont pas évidentes », même si nous n'avons que les indices provenant des conséquences. 


\section{BIBLIOGRAPHIE}

Asher, J. 1969. « The total physical response approach to second language learning ». Modern Language Journal 53, 334-341.

Bailly, D. 1998. Les mots de la didactique des langues. Paris : Ophrys.

Berbaum, J. 1991. Développer la capacité d'apprendre. Paris : ESF.

Berbaum, J. 1992. Pour mieux apprendre. Paris : ESF.

Carr, P. 1993. Phonology. Londres : Macmillan.

Cyrulnik, B. 1983. Mémoire de singe et parole d'homme. Paris : Hachette.

Delannoy, C. 1994. Une mémoire pour apprendre. Paris : Hachette.

Ellis, R. 1985. Understanding Second Language Acquisition. Oxford : Oxford University Press.

Ellis, R. 1994. The Study of Second Language Acquisition. Oxford : Oxford University Press.

Freud, S. D. 1982 [1938]. Introduction à la psychanalyse. Paris : Payot.

Galisson, R. 1980. D’hier à aujourd'hui la didactique des langues. Paris : Clé International.

Gaonac'h, D. 1987. Théories d'apprentissage et acquisition d'une langue étrangère. Paris : Hatier.

Goleman, D. 1997. L'intelligence émotionnelle. Paris : Laffont.

Houssaye, J. 1992. Triangle pédagogique. Francfort : Peter Lang.

Hureau, M. \& E. Loarer. 1995. Peut-on éduquer l'intelligence? L'évaluation d'une méthode d'éducation cognitive. Francfort : Peter Lang.

Hyman, L. 1975. Phonology: Theory and Practice. New York : Holt.

Julié, K. 1994. Enseigner l'anglais. Paris : Hachette.

Jung, C. G. 1964. Essai d'exploration de l'inconscient. Paris : Gonthier.

Laborit, H. 1994. La légende des comportements. Paris : Flammarion.

Larsen-Freeman, D. \& M. H. Long. 1991. An Introduction to Second Language Acquisition Research. Londres : Longman.

Lass, R. 1984. Phonology, An Introduction to Basic Concepts. Cambridge : Cambridge University Press. Piaget, J. 1987 [1964]. Six études de psychologie. Paris : Folio Denoël.

Narcy, J-P. 1990. Apprendre une langue étrangère. Paris : Éditions d'organisation.

Narcy, J-P. 1997. Didactique des langues, le kaléidoscope des théories. Strasbourg : $2^{\mathrm{e}}$ Colloque COFDELA.

O’Neil, C. 1993. Les enfants et l'enseignement des langues étrangères. Paris : Credif-Hatier-Didier.

Rolland, Y. 2002 [1999]. Storytelling et dénativisation phonologique à l'école élémentaire : une gageure didactique? Thèse de doctorat sous la direction de Jean-Paul Narcy. Villeneuve d'Asc : Septentrion Presses Universitaires.

Singleton, D. 1989. Language Acquisition: the Age Factor. Londres : Multilingual Matters. 
Thill, E. 1997. Motivation, émotion, attention et concentration. Paris : Vigot.

Trocmé-Fabre, H. 1992. J'apprends, donc je suis. Paris : Éd. d'organisation.

Troubetzkoy, N.S. 1986 [1949]. Principes de phonologie. Paris : Klincksieck.

Varela, F., E. Thomson \& E. Rosche. 1993. L'inscription corporelle de l'esprit. Paris : Seuil.

Widdowson, H. W. 1979. « Directions in the teaching of discourse ». In Brumfit, C. J. \& K. Johnson, The Communicative Approach to Teaching. Oxford : Oxford University Press.

\section{RÉSUMÉS}

Faire émerger une cohérence en didactique des langues est difficile face à des domaines de références souvent contradictoires. Les théories psychologiques axées sur la forme et le conditionnement ont précédé la psychologie cognitive, qui ne les a pas entièrement détrônées. Toutes ont influencé les théories d'apprentissage, le behaviorisme, le nativisme, l'environnementalisme, l'interactionnisme et l'approche heuristique. Les théories linguistiques ont aussi été modifiées selon les influences subies. La linguistique structurale formaliste, la linguistique et la parole, l'influence cognitiviste, la linguistique de l'énonciation ont toutes joué un rôle en didactique des langues. Les conflits déjà patents à ce niveau théorique se retrouvent aussi dans des fondements comme la notion d'âge, la dimension psychanalytique, le concept de phonème et la notion d'apprentissage. La nécessité de transcender ces fondements théoriques en élargissant vers d'autres champs semble nécessaire pour une plus grande cohérence didactique. Il en résulte une juxtaposition équilibriste d'approches certes contradictoires mais aussi nécessairement complémentaires.

Finding out a precise coherence in second language acquisition research is not easy when facing so many contradictory theoretical fields. Psychological theories based on conditioning and form were referred to before cognitive psychology appeared. But cognitivism did not completely eliminate previous theories. They all influenced learning theories, behaviorism, nativism, environmentalism, interactionism and the heuristic approach. Linguistic theories also changed. Structuralism, linguistics and speech, cognitivism, enunciation still all play an important role in second language acquisition. More conflicts can be found in concepts like the age factor, psychoanalysis, the phoneme concept and learning. It seems necessary to transcend these theoretical data and to accept to integrate new fields to achieve more coherence. The result is a delicate mixture of contradictory but necessarily complementary approaches.

\section{INDEX}

Mots-clés : apprentissage des langues, cohérence, conflit d'interprétation, éclectisme

Keywords : coherence, interpretation conflict, language learning, eclecticism

\section{AUTEUR}

\section{YVON ROLLAND}

Yvon Rolland est professeur des universités à l'université de La Réunion (FLSH-ESPE). Il est l'auteur d'une thèse sur l'importance des neurosciences pour la didactique de l'anglais et de 
plusieurs ouvrages sur l'apprentissage de l'anglais langue étrangère. yvon.rolland@univreunion.fr 